\title{
Prononciation, littérature et apprentissage du français langue étrangère avec les medias sociaux
}

\author{
Pronunciation, literature and learning French \\ as a foreign language with social media
}

\author{
MARio TOMÉ DíEz \\ Universidad de León \\ mtflenet@yahoo.es
}

\begin{abstract}
Résumé
La littérature peut fournir toute sorte de matériaux pédagogiques pour l'apprentissage de la prononciation du français langue étrangère (FLE), mais la pratique et la recherche dans ce domaine sont encore très peu développées. Les nouveaux outils et médias sociaux peuvent inverser cette tendance en favorisant la production orale des apprenants et en intégrant des tâches pédagogiques efficaces et motivantes. Depuis plusieurs années nous menons des recherches sur les applications des nouvelles technologies à l'enseignement / apprentissage du FLE à l'Université de León en Espagne et nous avons élaboré deux bases de données pionnières et complémentaires: une sur des ressources littéraires et l'autre sur les productions orales des étudiants. Nous abordons dans cet article le projet Littérature Française Multimédia FLE, une base de données audiovisuelle en ligne sur la littérature française pour l'enseignement et apprentissage du FLE et le projet Oral FLE Prononciation, un corpus oral des productions orales des apprenants espagnols de français langue étrangère.
\end{abstract}

\begin{abstract}
Literature can provide all kinds of teaching materials for learning pronunciation of French as a foreign language (FLE), but practice and research in this area are still very little developed. New tools and social media can reverse this trend by promoting the oral production of learners and by integrating effective and motivating teaching tasks. For several years we have been conducting research on the applications of new technologies to teaching / learning FLE at the University of León in Spain and we have developed two pioneering and complementary databases: one on literary resources and the other on students' oral productions. We discuss in this article the French Literature Multimedia FLE project, an online audiovisual database on French literature for teaching and learning FLE and the Oral FLE Pronunciation project, an oral corpus of oral productions of Spanish learners of French as a foreign language.
\end{abstract}


PronONCIATION, LitTÉRATURE ET APPRENTISSAGE DU FRANÇAIS LANGUE ÉTRANGÈRE AVEC LES...

\section{Mots-clés}

littérature française, médias sociaux, apprentissage de la prononciation, correction phonétique, Enseignement du français langue étrangère.

\section{Keywords}

french literature, social media, pronunciation learning, correctness of pronunciation, teaching of french as a foreign language.

\section{Introduction}

La littérature fournit des matériaux authentiques à la classe de Français Langue Étrangère (FLE) et les textes littéraires peuvent favoriser l'enseignement et l'apprentissage de la prononciation, contribuant ainsi au perfectionnement de la perception et de la production. Mais les recherches et les pratiques pédagogiques axées sur les apports de la littérature dans l'enseignement de la prononciation sont rares. C'est une orientation très fréquente en didactique des langues que de négliger l'acquisition des compétences orales, face au prestige et à la concurrence de la langue écrite.

Actuellement on dispose de directives éducatives, de méthodologies et de technologies pour améliorer cette situation, ainsi que pour favoriser spécialement l'acquisition des compétences orales grâce à la littérature. Dans cet article nous présentons les expériences et recherches sur le développement de la production orale et sur l'apprentissage de la prononciation à l'aide des ressources littéraires ainsi que des médias sociaux dans le cadre d'un dispositif de formation universitaire de FLE. Nous aborderons les implications pédagogiques suivantes:

- Des outils et médias sociaux pour le développement des compétences orales.

- Des tâches pédagogiques sur la littérature.

- Une base de données multimédia sur des ressources littéraires.

- Une base de données sur les productions orales des étudiants.

\section{Cadre théorique}

La littérature a occasionnellement fourni des matériaux authentiques à la classe de français, soit pour l'exploitation textuelle, soit par son approche culturelle ou interculturelle. Des revues et ouvrages spécialisés ont préconisé l'exploitation des textes littéraires dans l'apprentissage du Français Langue Étrangère: Benamou, 1979; Taillandier \& Bouchery, 2009; Tabaki-Iona et al., 2010; Pierré \& Treffandier, 2012; Fiévet, 2013; Defays et al., 2014; Aubert-Godard, 2015. Certes, ces publications traduisent un regain d'intérêt pour l'introduction du texte littéraire en classe de FLE, mais elles ne tiennent pas compte des potentiels pédagogiques pour l'enseignement et l'apprentissage de la prononciation.

Fredet \& Nikou (2020) dans un numéro monographie intitulé Phonétique, littérature et enseignement du FLE: théories et recherches rassemblent les recherches suivantes autour de cette problématique: 
Lauret se propose de construire les bases théoriques d'une exploitation phonétique du texte littéraire, tout en procédant à une identification des contenus phonétiques dont l'objectif principal est d'aider l'apprenant ou l'enseignant, à accéder au sens. Bourvon revient sur les difficultés des apprenants à lire et à déclamer les textes littéraires, difficultés dues à la fossilisation des compétences en lecture à voix haute acquises en langue maternelle et transférables, par la suite, en langue étrangère. Martin s'intéresse à l'intonation du FLE, en se fondant sur les découvertes en neurolinguistique qui portent sur la synchronisation de la perception des syllabes par les ondes cérébrales et il s'intéresse aux oscillations delta, thêta et gamma qui permettent de rendre compte de l'élaboration d'une structure prosodique du texte lu, de l'eurythmie des groupes accentuels et de l'alignement de ces groupes avec les unités syntaxiques. Pillot-Loiseau \& Schweitzer abordent l'étude de deux figures de style (assonance et allitération) et des figuralismes pour faire de la phonétique en classe de FLE, ainsi que leur apport à la pédagogie de la prononciation (en compréhension et en production) ou le rôle de l'expressivité du discours parlé et chanté.

Weber s'intéresse au statut et à la place de l'oralité, ainsi qu'à l'exploitation de la variation socio-linguistique dans les corpus littéraires du XXème siècle, qui offrent un terrain propice à la didactique de la prononciation du FLE. Auzéau réfléchit sur l'apport de l'utilisation du support dramatique (texte de théâtre), un outil pédagogique qui permet une approche participative et active (monter une pièce de théâtre) et qui met en lumière l'importance du travail de la voix et du corps (prononciation, intonation, position du corps, gestes, etc.). Blondel vient compléter cette réflexion en proposant certaines techniques d'acteur qui aident à surmonter les appréhensions, à apprendre à mieux articuler (mouvements des organes phonatoires) et à s'approprier les sons, les schémas intonatifs, le rythme de la langue étrangère. Cuet aborde l'apport des pratiques théâtrales pour l'amélioration de l'oral des étudiants chinois et constate que l'introduction de l'expression théâtrale en classe de FLE se révèle être une pratique dynamique pour travailler l'oral (exercices de communication et d'interaction) et corriger non seulement les erreurs phonétiques et prosodiques mais également transculturelles. Mousavishirazi s'intéresse à la beauté de la matière sonore des textes littéraires, de trois genres différents (théâtre; roman/autobiographie romancée; poésie), tout en se focalisant sur la nécessité de travailler conjointement la littérature et la phonétique et, plus particulièrement, de se tourner vers l'esthétique des sonorités du texte littéraire qui peut jouer un rôle important dans la motivation des apprenants.

Veldeman-Abry \& Abry se servent des exemples littéraires autour desquels sont construites des activités visant à sensibiliser les apprenants à la musicalité de manière à les aider à s'approprier les nouveaux sons, et par extension oralement le texte littéraire, par un travail de correction phonétique sur des phénomènes peu développés en classe de langue (le "e" muet, la diérèse, les enchaînements, etc.). Monville-Burston, Kakoyianni-Doa \& 
Armostis insistent sur l'importance de l'enseignement/apprentissage de la phonétique par l'intermédiaire d'une expérimentation, en cinq étapes et autour d'un poème d'Apollinaire. Caussade, Cornaz \& Yezhkova proposent des comptines à gestes pour la perception et la production des phonèmes et graphèmes du français, en se révélant comme des supports particulièrement propices à l'enseignement / apprentissage de la perception et de la production des phonèmes du français. Corona remarque que la poésie peut faciliter par ses sonorités, sa musicalité et sa matière / matérialité phonique, l'enseignement / apprentissage de la prononciation et de l'intonation. Patéli \& Nikou proposent deux textes: un poème de Verlaine et une nouvelle de Yourcenar pour travailler respectivement la compétence réception et la compétence lectorale, travail suivi de conseils / directives / instructions pédagogiques afin de guider les enseignants dans l'exploitation phonologique d'un texte littéraire. Bacor s'interroge sur l'apprentissage multisensoriel du rythme du français, s'appuyant sur des extraits filmiques (scénario cinématographique) qui en permettent l'appropriation visuelle. Enfin, Tomé s'appuie sur un dispositif de formation universitaire tout en abordant le rôle que les weblogs, les podcasts et les réseaux sociaux jouent dans le développement et l'optimisation de la compétence d'interaction orale ainsi que dans la correction de la prononciation par la littérature.

\section{Participants}

Les étudiants qui ont participé à cette recherche suivaient des matières de français à 1'Université de León, pendant la période 2005 à 2020: $2^{a}$ Lengua y su Littératura: Francés I; Lengua Francesa I et Lengua Francesa V: Grado en Filología Moderna: Inglés; Francés I: Grado en Comercio Internacional). Généralement leur niveau de langue était A1 ou A2 (Cadre européen commun de référence pour les langues) et leur âge entre 18 et 20 ans. Les tuteurs qui ont participé dans les projets de télécollaboration ou des échanges étaient habituellement des étudiants français (ERASMUS ou Master FLE) supervisés par un enseignant.

L'utilisation des blogs, podcasts, réseaux sociaux et de la plateforme Moodle faisait partie d'un dispositif de formation essentiellement présentiel (enseignant et apprenants dans la salle d'ordinateurs) et occasionnellement à distance pour les travaux d'enregistrement audio, ainsi que pour les tâches qui appartenaient aux projets de télécollaboration León-Grenoble et Echanges Lille-Léon. Les expérimentations étaient axées sur l'apprentissage de la prononciation et spécialement sur les productions orales des apprenants, qui ont été enregistrées sur différents supports informatiques (fichiers mp3 ou wav, podcasts ou audioblogs en ligne, séquences vidéo en format flv ou avi). 


\section{Outils et médias sociaux pour la production orale}

Pendant la période 2005 à 2020 on a testé et sélectionné différents médias sociaux ainsi que la plate-forme e-Learning Moodle. On a privilégié les critères suivants dans leur utilisation et sélection:

- Intégration d'un magnétophone pour l'enregistrement des séquences audio.

- Fluidité et facilité dans la publication des productions orales ou écrites.

- Consultation facile et dynamique des podcasts ou messages écrits.

- Collaboration entre étudiants (écoute mutuelle, commentaires, corrections ou créations en commun).

Nous avons analysé le rôle de ces outils dans de diverses publications (Tomé 2007, 2009a, 2009b, 2011, 2015, 2016, 2017). Voici les plus importants et utiles pour notre recherche:

Blogg.org, Dotclear, Canalblog, Overblog sont des serveurs de weblogs qui ont été utilisés comme des blogs des étudiants ou comme blog de classe pour effectuer diverses tâches de compréhension orale ou de production écrite. Loudblog, système de gestion de contenu (CMS), qui intègre des podcasts a été installé sur le serveur de l'Université de León et a permis la création du blog Audio Blog - Campus Virtual FLE. Ces dernières années, nous avons essayé Wikispaces, un service d'hébergement de wikis, pour coordonner les podcasts des étudiants, ainsi que pour préparer l'évaluation des productions orales.

Podomatic est un serveur de podcasts qui a permis la création de messages écrits accompagnés de séquences audio. Les premiers enregistrements audio ont été réalisés dans le podcast Audio Blog FLE 2005-06.

AudioBoom, Soundcloud, Twaud.io et Jamglue sont devenus les espaces de production orale des étudiants. Ces médias sociaux ont un magnétophone web qui permet la publication rapide de messages audio. UStream.tv est une communauté de broadcasting qui a facilité l'enregistrement de séquences vidéo dans le cadre des projets de télécollaboration.

La plateforme Moodle a été l`espace de communication du projet de télécollaboration Léon-Grenoble; les tuteurs grenoblois et étudiants de León ont utilisé les Forums pour réaliser les différentes tâches écrites, ainsi que pour l>échange de fichiers audio et vidéo. Le réseau social Ning a permis les interactions du projet Echanges León-Lille; ainsi que des collaborations pour la correction de prononciation entre les étudiants ERASMUS natifs français et les étudiants de l'Université de León. 


\section{Des tâches pédagogiques sur la littérature}

Pour notre recherche nous avons élaboré et essayé différentes modalités de tâches pédagogiques: exercices, dictées, cybeRallyes, projets (Tomé, 2007, 2009a, 2009b, 2011, 2016); et en relation avec apprentissage de la prononciation nous nous sommes centrés sur les objectifs suivants:

- Développer les compétences de production et compréhension orales chez les apprenants.

- Faciliter des contextes réels de communication et de collaboration.

- Favoriser la correction de la prononciation entre apprenants, tuteurs et enseignants.

Le modèle de tâche pédagogique est composé généralement des éléments suivants:

- Instructions claires et organisées par étapes.

- Ressources web (textes, sites et matériels audiovisuels).

- Séquences audio ou vidéo en relation avec la compréhension orale.

- Production orale avec plusieurs modalités d'enregistrement : Podcasts (AudioBoo, Soundcloud), magnétophone du PC ou du téléphone portable.

- Pistes, informations ou outils pour accompagner et aider l'apprenant.

Exemple: Tâche audiovisuelle Littérature: Baudelaire sur Loudblog: Campus Virtuel FLE - Audio Vidéo Blog:

\section{Tâche Production Orale: A la découverte de la littérature française:}

\section{Charles Baudelaire (1821-1867)}

\section{INSTRUCTIONS:}

1. Nous allons consulter la biographie et les poèmes sur le site: Wikipédia: Baudelaire

2. Visitez le site Baudelaire Audio Vidéo pour regarder les vidéo activités.

3. Sélectionnez un poème sur le site Wikisource: Les fleurs du mal

4. Enregistrez le poème sur votre espace "Jamglue"

5. Préparez votre production orale en écoutant le poème sur le site:

Fleurs du mal-Audio poèmes

6. Déposez le texte du poème et le lien audio dans les "Commentaires". 
Cliquez sur l'image pour voir la vidéo

Pistes et outils complémentaires:

Littérature Ressources FLENET

Audio Vidéo Littérature - Baudelaire. <http://flenet.unileon.es/docauteurs.html\#Baudelaire>

Outils: Dictionnaires - Correcteurs

Grammaire Audiovisuelle FLE < http://flenet.unileon.es/grammairecours.htm>

Lexique Audiovisuel FLE <http://flenet.unileon.es/lexiquecours.htm>

Phonétique Française FLE <http://flenet.unileon.es/phon/indexphon.html>

Voici quelques modalités de tâches pédagogiques que l'on peut consulter sur les médias sociaux, ainsi que sur la base de données Projet Oral FLE Prononciation:

A. Tâches interculturelles et de compréhension orale:

- Vidéo Activités Pédagogiques. Des courts documentaires en version vidéo sur des aspects de la culture et la littérature françaises: Stendhal et Grenoble, Le Petit Prince, Baudelaire: Hymne à la beauté, Corneille Actualités, Stendhal Exposition, Nothomb Mystères, Découvertes Astérix et Tintin, La Marseillaise - Compréhension Orale.

- Textes Audio - FLENET. Des extraits de romans en version audio avec ses textes: Camus, L'étranger; Perrault, Le Petit Poucet; Maupassant, Le Horla; Vian, L'écume des jours; Stendhal, Le Rouge et le Noir; Zola, Germinal; Chateaubriand, René; B.de Saint-Pierre, Paul et Virginie.

- Dictées multimédia autocorrectives. Des textes littéraires en version audio avec la transcription pour la correction de la dictée: Daudet, La chèvre de M. Seguin; Saint-Exupéry, Terres des hommes; Anouilh, La guerre de Troie n'aura pas lieu; Barrès, Greco ou le Secret de Tolède; Proust, Du côté de chez Swann.

B. Tâches de production orale et correction de la prononciation:

- Lecture de textes littéraires. Les Textes Audio - FLENET du chapitre antérieur ont été conçus pour cette intention, ainsi que la vidéo activité Baudelaire: Hymne à la beauté ou la Tâche PO et PE: Visite Bibliothèque virtuelle.

- Tâches multimédia pour la production orale. Elles présentent les caractéristiques et objectifs que nous avons indiqué plus haut, en suivant le modèle de la TPO: Le Petit Prince: TPO: A la découverte de la littérature française: Baudelaire; CybeRallye FLE: Jules Verne; A la découverte de la chanson française; Vendredi ou la vie sauvage - Tâches Orales. 
- Exercices de répétition ou sensibilisation aux phonèmes, mots ou chansons: des onomatopées, des cris, des virelangues ou des chansons pour l'exploitation et le renforcement des phonèmes problématiques: jeux, cris et chansons. Les étudiants chantent des chansons.

- Corrections collaboratives, jeux, théâtralisations et créations (TPO: Le Petit Prince; Correction collaborative Prononciation - Vidéo Tâches).

\section{La base de données Littérature Multimédia FLE}

Dans le cadre du projet FLENET (Français langue étrangère et Internet) l'Université de León propose depuis 1999 une base de données audio et vidéo à destination des étudiants, des enseignants et des chercheurs en français langue étrangère.

Plus d'une centaine d'auteurs sont répertoriés dans la base de données Littérature Multimédia FLE. Un index des noms permet de se retrouver plus rapidement dans le corpus proposé. Des entrées sont également créées pour certains genres et périodes spécifiques de l'Histoire littéraire: Moyen-Âge, XXème, XXIème siècle, romantisme, surréalisme.

Les objectifs de ce vaste projet qui est actualisé périodiquement sont les suivants:

- Privilégier la compréhension orale pour l'approche des œuvres ou des auteurs.

- Fournir aux enseignants un fond sonore pour la classe de FLE ou de littérature.

- Favoriser chez les étudiants la découverte et le contact avec la littérature.

- Proposer des tâches pour l'acquisition des compétences orales chez les apprenants de FLE.

- Elaborer une histoire audiovisuelle de la littérature française ou francophone.

Nous trouvons dans cette base de données des ressources très variées:

A. Des fichiers audio et vidéo consacrés aux grands écrivains de la langue française. D'Apollinaire à Zola, en passant par Camus, Nerval ou Yourcenar, on peut consulter, écouter ou regarder de nombreux documents audio et vidéo sur les grands auteurs de l'histoire de la littérature française:

Honoré de Balzac: Ressources Audio - Internet Archive

Charles Baudelaire: Une vie, une oeuvre - France Culture

Camus, lumières et ombres - Dailymotion

Chateaubriand Vidéos - Archives RSR

Vidéo: Découverte Corneille - Le Cid

Gustave Flaubert Audiovisuel - Campus Education

Jean Giono - INA vidéos Recherche 
B. Des audio livres sur les ouvrages de l'histoire de la littérature. Un livre audio est un livre ou un texte dont on a enregistré la lecture à haute voix. Le livre audio est d'abord créé au XXème siècle pour la jeunesse (histoires pour les enfants, méthodes de langues pour le parascolaire) et pour un public de malvoyants. Un bon nombre de pièces et de textes sont lus et enregistrés à la radio. Actuellement les progrès de la synthèse vocale permettent de produire des livres en recourant à cette technique. Les nouveaux médias sociaux ont favorisé le développement des livres audio et l'on trouve de nombreuses ressources sur le web actuel:

\section{Les Misérables de Victor Hugo - Fueilleton France Culture}

La leçon - La cantatrice chauve - UbuWeb

Les Caractères, La Bruyère-Audio livre-Audiocité.net

Fables de La Fontaine - LivriVox

Mallarmé - Poésies - Littérature audio.com

Maupassant-Livres audio gratuits

Archives Audio Molière - Canal Académie

C. Des conférences, cours ou séminaires sur la vie ou l'œuvre des écrivains. On dispose en ligne de beaucoup d'archives sonores sur les grands auteurs de la littérature française (Portails des institutions publiques: Bibliothèque nationale, INA, Collège de France, Canal $\mathrm{U}$; ainsi que les sites des universités ou des bibliothèques, etc.):

Montaigne ou l'homme tranquille - INA Video

Sylvie de Nerval et les genres lyriques - Canal U

Proust dans l'oeil des philosophes - Colloque - Diffusion ENS

Conférence J.P. Sartre: L'existentialisme est un humanisme

Conférences et cours sur Voltaire - E. Sonore

Vidéos H. Guillemin: E. Zola-Archives TSR

Aragon et l'Affiche rouge-Conférence F. Pras

D. Des émissions radio ou tv consacrées à la littérature (interviews, dossiers, magazines). La littérature est un thème présent dans les programmes de radio et de télévision depuis leurs origines. Les nouveaux médias sociaux poursuivent cette orientation avec des réflexions, des débats ou des émissions sur différents aspects de la vie littéraire actuelle ou sur l'histoire de la littérature française ou francophone:

Antonin Artaud - France Culture

Blaise Cendrars au firmament - RTS Archives

Emissions Patrick Modiano - France Inter

Le long chemin d'Henri Troyat-Radio Canada 
Anales de Filología Francesa, n. ${ }^{\circ}$ 28, 2020

PRONONCIATION, LITTÉRATURE ET APPRENTISSAGE DU FRANÇAIS LANGUE ÉTRANGÈRE AVEC LES...

L'humeur vagabonde: Apollinaire - Espace2 RTS

Baudelaire: Une vie, une oeuvre-France Culture

Boris Vian - Audioblogs ARTE Radio

E. Des livres en ligne ou des extraits des œuvres littéraires. Le web actuel nous permet d'accéder à toute sorte de textes littéraires numériques en différents formats (Gallica, Abu, Anthologie sonore de poésie française, Athena, Wikisource, Babelio, Bibliothèque électronique de Lisieux, Gutenberg.org, Internet Archive, etc.):

Oeuvres de Balzac - Gallica BNF

L'écume des jours - Ebook en ligne

Le comte de Monte-Cristo - Internet Archive

Flaubert - Oeuvres - Bibliothèque ABU

Oeuvres d'Apollinaire - Wikisource

Bibliothèque nationale de France - Victor Hugo

Fables de la Fontaine - Les grands classiques

F. Des tâches multimédia pour la découverte ou l'exploitation pédagogique des ouvrages ou des auteurs de la littérature française. De nombreuses ressources numériques nous proposent des exercices ou des activités pédagogiques sur la vie ou l'œuvre des grands écrivains de l'histoire de la littérature française (dictées autocorrectives, cybeRallyes, webquests, exercices audiovisuels, etc):

Le Petit Prince TPO-Activité Orale

Tâche audiovisuelle Littérature: Baudelaire

Littérature: un écrivain, une oeuvre

Dictée autocorrective - Martinville - M.Proust

CybeRallye FLE - Jules Verne

Stendhal:Littérature et politique - VideoActivité

Baudelaire "Hymne à la beaute" - VideoActivité

G. Des sites web, bibliographies et dossiers sur les œuvres ou les auteurs. Les écrivains et les ouvrages littéraires sont présents sur Internet et les nouveaux médias sociaux permettent une grande diffusion de contenus numériques et multimédia de toute sorte: portails et sites web sur les auteurs, expositions multimédia, cours, thèses, colloques ou bibliographies, etc.):

Thèses sur Stendhal - Thèses.fr

Paul Valéry - Wikipédia 
Anales de Filología Francesa, n. ${ }^{\circ}$ 28, 2020

MARIo ToMÉ DíEz

Association Les amis de Paul Verlaine

Voltaire Foundation - University of Oxford

Exposition et Dossier Zola - BNF

Marcel Pagnol-Académie Française

Fonds Michel Tournier - Université d'Angers

\section{Le corpus Oral FLE Prononciation.}

Le Projet Oral FLE - Prononciation constitue une base de données des productions orales des apprenants de FLE, élaborée dans le cadre des expérimentations réalisées par le Campus Virtuel FLE et par le Projet FLENET de l'Université de León et de FLENET RedIRIS (Red Académica y Científica española). Les objectifs fondamentaux de ce corpus oral sont les suivants:

- Constituer un fond sonore à partir des enregistrements audio et des productions orales des étudiants de FLE déposés dans les podcasts, weblogs, réseaux sociaux et plate-forme de formation (Moodle).

- Classifier et analyser ces productions orales dans le contexte éducatif de l'enseignement / apprentissage de la prononciation du français langue étrangère.

- Proposer à la communauté scientifique du FLE les résultats et les expériences de ces recherches.

Dans le projet Oral FLE Prononciation les outils et applications du podcasting ou baladodiffusion (weblogs, podcasts, lecteurs multimédia, magnétophones web) ont joué un rôle très important, parce que la plupart des enregistrements audiovisuels ont été réalisés à l'aide de ces technologies ou médias sociaux. Les premiers enregistrements de la production orale publiés sur Internet, et expérience pionnière dans l'apprentissage des langues, ont été réalisés en octobre 2005 à l'université de León, par les apprenants d'un cours de FLE ( $2^{a}$ Lengua y su Literatura Francés I), dans le cadre du weblog Audio Carnet FLE 2005-06. Quelques auteurs (Belanger, 2005; McCarty, 2005) ont déjà remarqué que les premières applications du podcasting pour la compréhension orale ont eu lieu dans un cours d'anglais au Osaka Jogakuin College (Japon), ainsi que les premiers enregistrements audio (sur des iPods) ont eu lieu dans un cours d'espagnol au printemps 2005 à l'Université de Duke (États-Unis). Malheureusement on ne conserve pas d'archives sonores de ces pratiques historiques.

Les séquences audiovisuelles du projet Oral FLE Prononciation nous a permis d'identifier et de compiler un système d'erreurs de prononciation des apprenants espagnols, qui n'était pas disponible jusqu'à présent. Les conséquences didactiques de ce système d'erreurs peuvent être déterminantes pour l'enseignement / apprentissage de la prononciation et pour la pratique de la correction phonétique. En relation avec cette pratique on a remarqué en même temps plusieurs indicateurs qui se sont révélés comme des indices ou des facteurs 
déterminants pour l'évaluation de la production orale, ainsi que pour l'amélioration de la production.

Dans le processus de correction phonétique l'application de méthodologies par les apprenants se révèle aussi déterminante. Dans notre recherche nous les avons déjà analysées dans des publications précédentes (Tomé, 2015, 2016, 2017, 2020). Parmi les méthodes de correction de la prononciation nous avons remarqué les modalités suivantes: Répétitions tutorisées, autoréflexions, corrections collaborative, ainsi que l'application des techniques de correction suivantes: échauffements vocaux, autocorrections, répétitions, renforcements, explications linguistiques.

A. Répétitions tutorisées par un enseignant, pair ou tuteur. Comme l'apprenant doit répéter un enregistrement il a besoin de répérer l'erreur, faire une réflexion et procéder à des entraînements. Le nouvel enregistrement peut être ainsi accompagné de différentes techniques ou strategies de correction de la prononciation. On a constaté que ces répétitions tutorisées impliquaient une amélioration dans les problèmes d'articulation ainsi que pour l'acquisition d'une prononciation correcte. Dans la base de données Projet Oral FLE Prononciation on peut trouver plusieurs de ces échantillons dans les rubriques ou dossiers suivants:

Etublog05_2006/:Dani_Elena_vache.mp3, David_Olga_sonZ.mp3, lena_Dani_sonY. mp3, Lidia_Marta_sonCH.mp3.

LitteraturePhon: Maria_echauff_3chNasales.m4a, Irene_chLoup_ErrorY.m4a, Sandra_EAchLoup.m4a.

B. Autoréflexion de l'apprenant dans la découverte de ses difficultés articulatoires et de ses erreurs de prononciation. L'enseignant indique un exercice ou séquence dans laquelle l'apprenant doit trouver les fautes de prononciation et les noter dans son cahier. Les passages des enregistrement audio qui contiennent des autocorrections revèlent spécialement ce facteur si déterminant pour l'amélioration et correction de la prononciation. Nous trouvons des exemples d'autocorrections dans les suivants dossiers et fichiers audio du Projet Oral FLE Prononciation:

Twaudio2010/: AutoCo_sonZL1E7EstefaniaModele.mp3, AutoCo_sonYL7e4Rebeca.mp3, AutoCo_sonCHL3e4Sofia.mp3.

LitteraturePhon: 2voix_Auclairlune.mp3, Carla_chPP_ErrYod.wav.

C. Application de méthodes et techniques de correction en autonomie ou en collaboration. On a pu observer dans les podcasts des étudiants l'effort articulatoire mis en pratique dans les répétitions succésives d'un phonème, mot ou phrase, dans l'exagération ou renforcement d'un phonème, dans les bruits ou onomatopées, dans les entraînements avec échauffement vocal ou dans différentes formes de théâtralisation créées par les apprenants. On peut consulter plusieurs exemples dans le Projet Oral FLE Prononciation: 
Twaudio2010/: Echauffement_nasaleSandra.mp3,EA_sonOE_L2E1Virginia.mp3, EA_sonJ_L3E3Jessica.mp3, VCorrige_L4E1Cristina.mp3.

LitteraturePhon: Maria_echauff_3chNasales.m4a, Irene_chLoup_ErrorY.m4a, Sandra_EAchLoup.m4a, 2voix_CCauclairlune.mp3, CO_RaquelJudith_poeme.mp3, TO_LorenachevreMSeguin.mp3.

D. Correction collaborative entre étudiants. Les enregistrements d'entraide impliquaient une préparation et réflexion préalables, l'application de stratégies de correction effectives, ainsi qu'une simulation de l'interaction entre l'enseignant (tuteur) et l'apprenant. Lorsque les étudiants réalisaient cette sorte d'enregistrements ils obtenaient habituellement une amélioration dans la correction des erreurs de prononciation. Ainsi nous proposons les exemples suivants de la base de données Projet Oral FLE Prononciation:

Twaudio2010/: Collab_L5E3_VirginiaRebeca.mp3, JuanAlba_TaxiL1.mp3, ChansonFJacques_LauraVirginiaSamuel.mp3.

LitteraturePhon: Col_LaBelleEpoqueMaraLauraMnica.mp3, 2voix_Auclairlune. mp3, 3voix_BonjourMaCousine.mp3.

\section{Conclusions}

Les potentiels de la littérature pour l'enseignement/apprentissage de la prononciation et pour la correction phonétique restent encore à explorer, spécialement dans l'élaboration de nouvelles ressources didactiques en classe de FLE. La base de données Littérature Multimédia FLE devra s'actualiser et enrichir avec de nouvelles ressources audiovisuelles. La création de nouvelles tâches pédagogiques sur la littérature devra être prioritaire, tout particulièrement en relation avec les tâches pour la production orale et pour l'apprentissage de la prononciation. Il serait important de conjuguer les nouvelles technologies et les médias sociaux avec des tâches efficaces et motivantes qui actualiseraient des situations réelles de communication et de collaboration chez les étudiants. Cette base de données multimédia en ligne pourrait en même temps devenir une audiovidéothèque de l'histoire de la littérature française, et compléter ainsi le grand patrimoine des ressources écrites sur la littérature que l'on trouve sur internet.

Les applications et potentiels pédagogiques du projet Oral FLE Prononciation restent ouverts à la communauté scientifique et académique et il devra se développer et améliorer dans plusieurs aspects. Le site web devrait être élargi dans plusieurs directions: sélection et publication de nouveaux enregistrements audiovisuels; création de nouvelles sections en relation avec le système d'erreurs, ainsi que de leur classification; organisation d'une typologie sur les différentes méthodes de correction pratiquées par le étudiants, tuteurs et enseignant; 
PronONCIATION, LitTÉRATURE ET APPRENTISSAGE DU FRANÇAIS LANGUE ÉTRANGÈRE AVEC LES...

amélioration des transcriptions orthographiques de tous les fichiers sonores; étude du traitement des problèmes liés aux aspects suprasegmentaux (prosodie, rythme et intonation); estimation de la fréquence des erreurs et techniques de correction; codage et étiquetage des séquences audiovisuelles avec l'application de programmes d'analyse de la parole. Il y a donc un long chemin à parcourir, mais toutes les améliorations futures renforceront l'objectif principal et l'origine du projet, qui est d'offrir aux apprenants et aux enseignants de FLE un corpus des productions orales dans le domaine de la recherche et de la pratique de la prononciation du français langue étrangère.

\section{Références bibliographiques}

Aubert-Godard, Anne. 2015. La littérature dans l'enseignement du FLE. Paris. Didier.

Belanger, Yvonne. 2005. Duke University iPod first year experience final evaluation report. Durham: Duke University.

Benamou, Michel. 1979. Pour une nouvelle pédagogie du texte littéraire. Paris, Hachette.

Defays, Jean-Marc, Delbard, Olivier \& Sarra Hammami. 2014. La Littérature en classe de FLE: états de lieux et nouvelles perspectives. Paris, Hachette.

FIÉvet, Martine. 2013. Littérature en classe de FLE. Paris, CLE International.

Fredet, Florentina \& Christos Nikou (dir.). 2020. "Phonétique, littérature et enseignement du FLE: théories et recherches" in CORELA (Cognition, Représentation, Langage), HS 30. $<$ http://journals.openedition.org/corela/9987> [22/05/2020].

MCARTY, Steve. 2005. "Spoken Internet To Go: Popularization through Podcasting". The JALT CALL Journal, 1(2), 67-74. <http://journal.jaltcall.org/articles/1_2_McCarty.pdf> $[12 / 05 / 2020]$.

Pierré, Marjolaine \& Frédérique TrefFAndier. 2012. Jeux de théâtre. Grenoble. PUG.

Tabaki-Iona, Frédérique, Proscolli, Argyro \& Kyriakos Forakis (dir.). 2010. La place de la littérature dans l'enseignement du FLE. Actes du Colloque international d'Athènes. Presses de l'Université d'Athènes.

TaILAndier, Isabelle \& Caroline Bouchery. 2009. Le FLE par les textes: Littérature et activités de langue. Paris, Belin.

Tomé, Mario. 2007. "Expériences pédagogiques dans le Campus Virtuel FLE” in ELA: Etudes de Linguistique Appliquée: Revue de didactologie des langues-cultures et de lexiculturologie; D'autres espaces pour les cultures. $\mathrm{n}^{\mathrm{o}} 146,179-188 .<\mathrm{http}$ ://www.cairn.info/ revue-ela-2007-2-page-179.htm $>$ [20/05/2020].

Tomé, Mario. 2009a. "Compétences orales et outils de communication Web dans un projet de télécollaboration pour l'apprentissage du français langue étrangère" in The Journal of Distance Education, vol. 23, $\mathrm{n}^{\circ}$. 1, 107-126. <http://www.jofde.ca/index.php/jde/article/ view/578/837> [14/05/2020]. 
Tomé, Mario. 2009b. "Productions orales, weblogs et projet de télécollaboration avec le web 2.0 pour l'enseignement du français (FLE)" in ALSIC (Apprentissage des Langues et Systèmes d'Information et de Communication), vol. 12, 90-108<https://journals.openedition. org/alsic/1279> [20/05/2020].

Tomé, Mario. 2011. "Réseaux et médias sociaux sur internet pour l'apprentissage de la prononciation d'une langue étrangère" in The Journal of Distance Education, vol. 25, no .2. $<$ http://www.jofde.ca/index.php/jde/article/view/724/1261> [20/05/2020].

TомÉ, Mario. 2015. "Development, analysis and evaluation of oral production in foreign language class with new technologies" in Thélème. Revista Complutense de Estudios Franceses, Vol. 30, 2: 255-268. <http://revistas.ucm.es/index.php/THEL/article/view/48663/47215> $[11 / 05 / 2020]$.

Tomé, Mario. 2016. “Compétences orales et nouvelles technologies dans un cours de français langue étrangère" in Çédille. Revista de estudios franceses, $\mathrm{n}^{\mathrm{o}} 12,387-401$. <http:// cedille.webs.ull.es/12/20tome.pdf> [13/05/2020].

Tomé, Mario. 2017. “Correction de la prononciation avec les médias sociaux dans l'apprentissage du français langue étrangère" in Synergies Europe, ${ }^{\circ} 12,107-123$. <http://gerflint.fr/ Base/Europe12/Tome.pdf> [20/05/2020].

Tomé, Mario. 2020. “Técnicas de corrección de la pronunciación en Internet para el aprendizaje del Francés lengua extranjera" in Thélème. Revista Complutense de Estudios Franceses, 35(1): 77-85.

Litterature Française Multimédia. Universidad de León. 2003. <http://flenet.unileon. es/docauteurs.html> [20/05/2020].

Projet Flenet (Français langue étrangère et Internet). 1999. Universidad de León <http:// flenet.unileon.es/> [20/05/2020].

Projet Echanges León-Lille. 2009-2010. <http://flenet.rediris.es/ProjetLeonLille/indexPLL.htm> [20/05/2020].

Projet León - Grenoble. 2005-2009. <http://flenet.rediris.es/projetLG/webProjetLG05. htm> [20/05/2020].

Projet Oral Fle Prononciation. 2006. Universidad de León < http://flenet.rediris.es/ OralFLEprojet/index.htm> [11/05/2020]. 
